\title{
MiCHEL PASTOUREAU
}

\section{Le roi du jeu d'échecs $\left(\mathrm{X}^{\mathrm{e}}-\mathrm{XIV}^{\mathrm{e}}\right.$ siècle $)$}

Le plus ancien texte occidental qui mentionne le jeu d'échecs date du début du $\mathrm{XI}^{\mathrm{e}}$ siècle: dans un acte daté de 1008, le comte d'Urgel Ermengaud $\mathrm{I}^{\mathrm{er}}$ lègue le jeu d'échecs qu'il possède à l'église de Saint-Gilles'. Quelques décennies plus tard, en 1061, le grand théologien Pierre Damien, alors cardinal d'Ostie, dénonce au pape l'évêque de Florence, qu'il aurait vu jouer aux échecs². Ce faisant, il inaugure la longue suite de diatribes par lesquelles l'Église, presque jusqu'à la fin du Moyen Âge, a condamné ce jeu. En vain. À partir du siècle suivant, les témoignages textuels, archéologiques et iconographiques se multiplient, qui soulignent combien, malgré l'hostilité de l'Église, le jeu d'échecs s'est rapidement répandu. Les princes et les prélats ne sont plus les seuls à s'y adonner; on y joue désormais dans toute l'aristocratie et dans tous les pays de la chrétienté, de la Sicile à l'Islande.

Ce sont les Arabes qui ont transmis les échecs aux Occidentaux. La pénétration s'est faite par une double voie; d'abord, peut-être dès le milieu du $\mathrm{X}^{\mathrm{e}}$ siècle, par une voie méditerranéenne: Sicile, Italie du Sud, Espagne (d'où leur première mention dans un texte catalan); ensuite, quelques décennies plus tard, par une voie septentrionale, les Scandinaves, qui commerçaient en Ukraine et sur les bords des mers Noire et Caspienne, ayant rapporté vers le nord l'usage de ce jeu pratiqué depuis plus de trois siècles en Inde du Nord, son berceau d'origine, puis en Iran et enfin en pays d'Islam. Les trouvailles archéologiques témoignent de ce double itinéraire et de l'occidentalisation progressive du jeu et des pièces ${ }^{3}$.

Lorsque l'Islam transmet le jeu d'échecs aux Occidentaux vers le milieu ou la fin du $\mathrm{X}^{\mathrm{e}}$ siècle, ces derniers ne savent pas jouer. Non seulement ils ne savent pas jouer, mais, lorsqu'ils essayent d'apprendre, ils sont déroutés par les principes du jeu, par la nature et la marche des pièces, par l'opposition des couleurs (camp rouge contre camp noir) et même par la structure de l'échiquier: soixante quatre cases, cela ne représente rien, ou peu de chose, dans la symbolique chrétienne des nombres. Les échecs sont un jeu oriental, né en Inde, transformé en Perse, remodelé par la culture arabe. Mis à part sa parenté symbolique avec l'art militaire, tout ou presque y est étranger aux chrétiens de l'an mille. Il faut donc pour assimiler ce jeu nouveau le repenser en profondeur, l'adapter aux mentalités occidentales, lui donner une image plus conforme aux structures de la société féodale 4 . Cela prit sans doute de longues décennies et explique que les

1 Harold J. Murray, A History of Chess, Oxford 1913, p. 405-407; Richard EALES, Chess. The History of a Game, London 1985, p. 42-43.

2 Murray, A History of Chess (voir n. 1), p. 408-415.

3 Michel Pastoureau, L'arrivée du jeu d'échecs en Occident. Histoire d'une acculturation difficile, dans: ID., Une histoire symbolique du Moyen Âge occidental, Paris 2004, p. 269-292.

4 Ibid., p. 279-283. 
textes, narratifs ou littéraires, qui aux $\mathrm{XI}^{\mathrm{e}}, \mathrm{XII}^{\mathrm{e}}$ et $\mathrm{XIII}{ }^{\mathrm{e}}$ siècles parlent du jeu d'échecs soient si confus, si contradictoires, si imprécis 5 .

Ce qui déconcerte d'abord les Occidentaux, c'est le déroulement même de la partie et son but final: rechercher la victoire et faire en sorte que le roi adverse soit en position de »mat«, c'est à dire incapable de bouger, comme un prisonnier ou, mieux, comme un mort. Une telle pratique est contraire aux habitudes de la guerre féodale, où les rois et les princes doivent se déplacer constamment: nobilitas/mobilitas, proclame déjà un jeu de mots qui traversera tout le Moyen Âge ${ }^{6}$. En outre, les rois ne sont pas faits pour être capturés, et les combats n'ont pas vraiment d'issue, ni dans un sens ni dans un autre. On s'arrête quand vient la nuit ou quand vient l'hiver, ou encore quand cesse la durée de l'host, mais pas quand l'adversaire est mis en déroute. Se battre compte alors beaucoup plus que gagner.

Il n'en va pas de même sur l'échiquier, où il faut qu'il y ait un vainqueur et un vaincu. En fait, la partie d'échecs telle qu'elle arrive en Occident ressemble plus à une bataille qu'à la guerre. Or, ce sont deux choses très différentes pour les mentalités et les habitudes féodales. Dans l'Occident des $\mathrm{XI}^{\mathrm{e}}$ et $\mathrm{XII}^{\mathrm{e}}$ siècles, les batailles véritables sont rares. Elles ont un statut bien particulier, remplissent une fonction proche de l'ordalie et se déroulent selon un rituel presque liturgique ${ }^{7}$. La guerre, au contraire, est faite d'incessants combats de petits groupes, de harcèlements répétés, d'escarmouches infructueuses, de chevauchées incertaines, de recherches de butin. C'est un rituel d'une autre nature, qui constitue la vie quotidienne et la raison d'être du seigneur et de ses chevaliers. La guerre n'a rien de liturgique, ni rien d'une sanction divine, comme c'est le cas de la bataille. Elle ne s'apparente donc guère à une partie d'échecs. Ses enjeux sont plus minces et plus diffus, et les rois n'y jouent guère un rôle de premier plan.

$\mathrm{Au} \mathrm{XIII}{ }^{\mathrm{e}}$ siècle, toutefois, cette situation évolue. La lutte contre les infidèles a progressivement donné aux chrétiens l'habitude et le goût des batailles. En 1214 se déroule la première véritable grande bataille entre chrétiens: Bouvines. Pour la première fois, rois et princes sont au premier rang. Dès lors, la guerre féodale se transforme, les guerres snationales` et dynastiques apparaissent, et les rapports se font plus étroits entre le jeu d'échecs et les usages militaires ${ }^{8}$. Peu à peu, sur les champs de bataille, le comportement des rois, des princes et des chevaliers se modifie et prend un aspect quelque peu >échiquéen<. La tactique, sinon la stratégie, fait son apparition: comme aux échecs, protéger le roi pour l'empêcher d'être tué ou fait prisonnier devient un impératif. Au siècle suivant, Froissart, par exemple, nous apprend qu'en 1356, lors de

5 Fritz Strohmeyer, Das Schachspiel im Altfranzösischen, dans: Abhandlungen. Herrn Prof. Dr. Adolf Tobler, Halle 1895, p. 381-403; Pierre JONIN, La partie d'échecs dans l'épopée médiévale, dans: Mélanges de langue et de littérature du Moyen Âge et de la Renaissance, offerts à Jean Frappier par ses collègues, ses élèves et ses amis, Paris 1970, t. 1, p. 483-497.

6 Werner PARAVICINI, Interesse am Adel. Eine Einleitung, dans: ID., Otto Gerhard OEXLE (dir.), Nobilitas. Funktion und Repräsentation des Adels in Alteuropa, Göttingen, 1997, p. 11.

7 Sur la guerre et la bataille à l'époque féodale, voir Georges DUBY, Le dimanche de Bouvines, Paris 1973, p. 133-208.

8 Sur l'évolution de la guerre au Moyen Âge, voir Philippe CONTAMINE, La guerre au Moyen Âge, Paris ${ }^{6} 1999$. 
la bataille de Poitiers, six faux rois de France - c'est-à-dire six chevaliers habillés comme le roi, portant ses armoiries et suivis de sa bannière - étaient présents sur le champ de bataille. Ruse quelque peu grossière qui n'empêcha pas le roi Jean le Bon d'être fait prisonnier'.

Vers l'an mille, ce qui déroute également les Occidentaux lorsqu'ils reçoivent le jeu d'échecs du monde arabe, c'est la nature des pièces. En ce domaine il ne faut pas s'adapter mais transformer ${ }^{10}$. Des pièces arabo-persanes, seuls le roi (le chah, mot qui a donné naissance au nom même du jeu: scaccarius en latin, eschec en ancien français, Schach en allemand, etc.), le cavalier et le fantassin (pion) ne posent pas de problème. Ce n'est pas le cas du conseiller du roi, le vizir (firzan dans la terminologie arabe), que les Occidentaux conservèrent d'abord tel quel sous le nom latin de firtus (et sous le nom français vulgarisé de fierce), puis qu'ils transformèrent en reine dans le courant $\mathrm{du} \mathrm{XII}^{\mathrm{e}}$ siècle. Cette transformation s'opéra lentement, la métamorphose du vizir en reine n'étant définitive dans toute l'Europe occidentale que dans la première moitié du $\mathrm{XIII}^{\mathrm{e}}$ siècle. Ce changement atteste combien les pièces d'échecs christianisées étaient désormais davantage pensées comme formant sur l'échiquier une sorte de cour royale - voire une cour céleste - plutôt qu'une armée. Une difficulté cependant était apparue: un roi chrétien pouvait avoir plusieurs conseillers, mais il ne pouvait pas avoir plusieurs épouses; or, comme dans les règles actuelles, dès le $\mathrm{XII}^{\mathrm{e}}$ siècle la »promotion « des pions ayant atteint l'extrémité du camp adverse transformait ces pions en reines. Celles-ci avaient donc tendance à se multiplier sur l'échiquier. On prit donc l'habitude de qualifier de »dames" (dominae) les pions promus et de réserver l'appellation de »reine« (regina) à la seule pièce formant couple avec le roi, l'ancien fierce du jeu arabo-musulman ${ }^{11}$.

Il n'est pas impossible non plus que le modèle nouveau du couple royal, qui joue alors un rôle de plus en plus important dans les cours européennes du XIII ${ }^{\mathrm{e}}$ siècle, ait influencé le jeu d'échecs et contribué à transformer l'ancien vizir en reine et non pas en chancelier ou en sénéchal, comme cela aurait pu être le cas. Entre la société de cour et le jeu d'échecs les influences ont toujours été réciproques 12 .

Plus complexe que le cas du vizir est celui de l'éléphant. Dans le jeu indien d'origine, il incarnait pleinement l'armée, car l'éléphanterie y jouait un rôle de premier plan, remplaçant ou renforçant celui de la cavalerie. Les Arabes conservèrent l'éléphant, mais, comme ce fut le cas pour les autres pièces, ils le stylisèrent fortement, l'islam interdisant (en théorie) la représentation figurée des êtres animés. De l'éléphant ils ne gardèrent donc que les défenses, évoquées par deux sortes de protubérances cornues, surmontant un tronc massif. Les Occidentaux ne comprirent pas toujours cette pièce et lui firent subir des changements multiples. Soit ils s'appuyèrent sur le mot

9 David Nicolle, Poitiers, 1356. The Capture of a King, Oxford 2004. Voir aussi Françoise Beriac-Laine, Les prisonniers de la bataille de Poitiers (1356), Paris 2002.

10 Sur la transformation des pièces, voir Michel PAStoureau, L'échiquier de Charlemagne. Un jeu pour ne pas jouer, Paris 1990.

11 Jean-Michel MEHL, La reine de l'échiquier, dans: Marcel FAURE (dir.), Reines et princesses au Moyen Âge, Montpellier 2001, p. 323-331.

12 Jacques LE GofF, Saint Louis, Paris 1996, p. 281-284 et p. 729-734. 
arabe désignant l'éléphant, al fil, dont ils firent le latin alfinus, puis auphinus, et transformèrent l'éléphant en dauphin, en juge, en comte (ancien français: aufin), en arbre ou en porte-étendard (italien: albero et alfiere). Soit, plus fréquemment, ils se fondèrent sur la forme des protubérances cornues et y virent tantôt une mitre d'évêque, tantôt un bonnet de bouffon. Cette dualité s'est conservée jusqu'à nos jours, l'évêque s'étant maintenu dans les pays anglo-saxons et le fou s'étant largement imposé ailleurs ${ }^{13}$.

Quant au char du jeu musulman, il connut lui aussi des fortunes diverses. D'abord maintenu tel quel, il se transforma par la suite en chameau ou en animal exotique, puis en une véritable scène à deux personnages (Adam et Ėve, saint Michel tuant le dragon, deux chevaliers joutant). La tour n'a remplacé ces différentes figures que tardivement, au tournant du $\mathrm{XIV}^{\mathrm{e}}$ au $\mathrm{XV}^{\mathrm{e}}$ siècle, et pour des raisons qui ne sont pas encore totalement expliquées: peut-être a-t-on rapproché le mot latin rochus (terme désignant cette pièce et que l'on avait calqué sur l'arabe rukh [char]) du mot italien rocca désignant une forteresse? Quoi qu'il en soit, cette figure, le roc du jeu français, est demeurée instable jusqu'au $\mathrm{XV}^{\mathrm{e}}$ siècle ${ }^{14}$.

Mais revenons au roi. Sur l'échiquier il représente la pièce la plus importante, mais nullement la plus forte. Certes, il peut avancer dans toutes les directions, mais seulement d'une case en une case. Le roc (la tour), l'alfin (le fou), le fierce (la reine) et même le cavalier sont plus forts que lui. Chacun possède sa marche propre, mais tous peuvent avancer de deux ou plusieurs cases et même, sous certaines conditions, sauter par dessus les pions de leur propre camp, ce que le roi ne peut pas faire hormis à l'ouverture. En fait, dans la hiérarchie des différentes forces en présence, seul le pion est plus faible que le roi. En revanche, le roi constitue la pièce essentielle, puisque s'il est mis en position de »mat«, c'est-à-dire immobilisé, il est considéré comme mort et son camp a perdu. Il y a là une situation paradoxale, du moins selon nos logiques modernes: la pièce essentielle n'est pas la plus forte, tant s'en faut. À la fin du XII ${ }^{\mathrm{e}}$ siècle et tout au long du XIII ${ }^{\mathrm{e}}$, il est probable que cette contradiction apparente ne choquait guère, étant plus ou moins conforme aux usages de la guerre: le roi se devait d'être sur le champ de bataille, et même d'y donner l'exemple, mais il n'avait pas à se montrer le plus fort ni le plus efficace des combattants. Certains barons et chevaliers le supplantaient dans ce rôle qui n'était pas le sien. En outre, tout devait être fait pour éviter qu'il ne soit fait prisonnier; d'où une certaine position en retrait, à la fois nécessaire et pleinement royale ${ }^{15}$. La littérature en donne de nombreux exemples: aucun des grands romans arthuriens en prose de la première moitié du XIII ${ }^{\mathrm{e}}$ siècle ne nous montre le roi Arthur combattant plus vaillamment que les meilleurs des chevaliers de la Table ronde,

13 Sur cette pièce et ses transformations, voir PAstoureau, L'arrivée du jeu d'échecs (voir n. 3), p. $281-282$.

14 D'une manière générale, sur l'évolution des pièces, leur nature, leur nom, leur force et leur marche sur l'échiquier, voir MURRAY, A History of Chess (voir n. 1), p. 408-424; Jean-Michel MeHL, Les jeux au royaume de France, du XIII ${ }^{\mathrm{e}}$ siècle au début du XVI ${ }^{\mathrm{e}}$, Paris 1990, p. 76-97; PASTOUREAU, L'échiquier de Charlemagne (voir n. 10).

15 De ce point de vue, l'attitude de Philippe Auguste à Bouvines fut longtemps hésitante. Voir DuBY, Le dimanche de Bouvines (voir n. 7). 
Lancelot, Gauvain, Perceval et quelques autres ${ }^{16}$. De même, aucun chroniqueur français ne nous raconte qu'au cours de telle ou telle bataille Philippe Auguste, Louis VIII, Saint Louis ou Philippe III se serait montré le plus fort de tous les combattants. Il en va de même des chroniqueurs anglais ou allemands à propos des différents souverains d'Angleterre et d'Allemagne ${ }^{17}$. Un vrai roi n'est jamais le plus fort au combat. Ce n'est nullement son rôle ni sa mission.

Souvenons-nous également qu'au jeu d'échecs chaque camp représente à la fois une armée et une cour. Or, sur ce second terrain - la cour - un roi ne se définit pas par ses aptitudes militaires, mais par sa majesté et par la façon dont il est entouré, dont il prend conseil, dont il se comporte vis-à-vis de sa maisnie. Il en va pareillement sur l'échiquier: le roi n'est roi que parce qu'il fait couple avec une reine et qu'il est entouré de deux alfins, de deux cavaliers et de deux rocs. De même que le roi féodal se doit de prendre conseil de ses barons, de ses vassaux et de son entourage, de même le roi de l'échiquier ne >joueく pas seul, mais en liaison constante avec ces quatre autres pièces. Elles incarnent les personnages importants de la cour. Il n'est du reste pas impossible que l'alfin du jeu d'échecs, une fois transformé en fou dans les années 1300, ait servi de modèle au fou de cour, office pratiquement inconnu avant cette date dans les principales cours européennes, mais constamment présent dans celles de la fin du XIV et au $\mathrm{XV}^{\mathrm{e}}$ siècle ${ }^{18}$. Le jeu d'échecs n'a pas seulement été un miroir de la société curiale; il en a parfois aussi été une matrice.

Qu'elles soient en ivoire (éléphant, morse), en bois de cervidé, en os, en corne, en cristal de roche, en pierre ou simplement en bois, les pièces d'échecs médiévales se répartissent en deux catégories: celles qui sont figuratives et celles qui sont fortement stylisées. Ces dernières, héritées de la culture arabo-musulmane, qui interdit la figuration, annoncent déjà nos pièces modernes.

Lorsqu'il est figuratif, le roi ressemble à la plupart des autres images de roi en majesté que le Moyen Âge nous a laissées, notamment celles que montrent les sceaux des $\mathrm{XII}^{\mathrm{e}}$ et XIII ${ }^{\mathrm{e}}$ siècles ${ }^{19}$. Le roi échiquéen est ainsi le plus souvent représenté assis sur un trône, de face, vêtu d'un long manteau et tenant des objets symboliques dans ses mains (épée, sceptre, globe, fleuron); ses pieds sont placés sur un tapis ou sur des »degrés«, et il porte sur la tête une couronne plus ou moins haute. Aux échecs comme ailleurs, un roi en majesté, c'est toujours un personnage assis, vu de face, les mains pleines, la tête

16 Sur la fonction non guerrière du roi Arthur: Marie-Luce CHENERIE, Le chevalier errant dans les romans arthuriens en vers des $\mathrm{XII}^{\mathrm{e}}$ et XIII ${ }^{\mathrm{e}}$ siècles, Paris 1986, p. 51-56; Martin AuRELL, La légende du roi Arthur (550-1250), Paris 2007, passim et p. 333-364.

17 Une exception, cependant: Richard Cœur de Lion, roi hors du commun à tous points de vue. Voir John Gillenham, Richard Coeur de Lion. Kingship, Chivalry and War in the Twelfth Century, Londres, Rio Grande 1994; et Jean FLORI, Richard Cœur de Lion. Le roi-chevalier, Paris 1999.

18 Michel Pastoureau, Figures et couleurs. Études sur la symbolique et la sensibilité médiévales, Paris 1986, p. 23-34.

19 Otto Posse, Die Siegel der deutschen Kaiser und Könige von 751 zu 1806, Dresde 1909-1913; Martine DALAS, Corpus des sceaux français du Moyen Âge, t. 3: Les sceaux des rois et de régence, Paris 1991; Percy Ernst SchramM, Die deutschen Kaiser und Könige in Bildern ihrer Zeit (751-1190), nouvelle édition, Munich 1983. 
couronnée et les pieds reposant sur un sol qui n'est pas celui du commun des mortels ${ }^{20}$. Pour que la majesté soit complète, il faut que tous ces éléments de la figuration soient présents. C'est le cas au jeu d'échecs, où, en outre, le roi est toujours la pièce dont la taille est la plus haute et le poids le plus lourd. Le roi n'est certes pas le plus fort sur l'échiquier, mais c'est celui qui se voit le mieux, qui s'identifie le plus facilement, notamment parce qu'il est plus grand que toutes les autres pièces. Un roi se doit d'être grand. Non seulement symboliquement mais aussi physiquement. Au reste, la plupart des souverains d'Occident, du XI ${ }^{\mathrm{e}}$ au XIII ${ }^{\mathrm{e}}$ siècle, ont été corpulents ou de haute taille ${ }^{21}$.

Il en va de même pour ce qui concerne les pièces d'échecs non figuratives, celles qui sont stylisées géométriquement. Le roi y est le plus grand, le plus gros, le plus lourd, même si ce n'est pas lui le plus fort sur l'échiquier. La forme la plus fréquente est celle d'un cylindre surmonté d'une grosse boule, avec parfois une encoche bien nette dans le haut du cylindre. Seule la reine ressemble au roi, mais elle est de taille nettement plus petite. Les autres pièces sont différentes et pourvues de protubérances faisant saillies sur le cylindre: tête stylisée d'un cheval pour le cavalier; deux »cornes« plus ou moins écartées pour l'alfin devenu évêque ou fou; deux pointes en forme de créneaux arrondis pour le roc, future tour ${ }^{22}$. Aucune confusion n'est possible avec le roi.

Grand, rond, haut et lisse, le roi du jeu d'échecs conserve, même fortement schématisé, presque abstrait, toute sa majesté. Preuve que, au Moyen Âge central et finissant, la force guerrière et la majesté royale sont devenues deux »fonctions« très différentes, presque incompatibles.

20 On trouvera de belles reproductions de pièces d'échecs médiévales figurant le roi dans: Hans WichmanN, Siegfried WichmanN, Schach. Ursprung und Wandlung der Spielfiguren in zwölf Jahrhunderten, Munich 1960; F. Lanier GRAHAM, Chess Sets, New York 1968; Victor KEATS, The Illustrated Guide to World Chess Sets, New York 1985; Roswin FINKENZELLER, Wilhelm ZIEHR, Emil M. BÜHRER, Schach. 2000 Jahre. Das Spiel, die Geschichte, die Meisterpartien, Lucerne 1989; Coleen Schafroth, The Art of Chess, New York 2002.

21 Michel Pastoureau, Le temps des rois obèses $\left(\mathrm{XI}^{\mathrm{e}}-\mathrm{XIII}{ }^{\mathrm{e}}\right.$ siècle), dans: Agostino ParavicinI BAGLIANI (dir.), La Mesure (Micrologus, 14), sous presse.

22 On trouvera des reproductions des pièces stylisées médiévales dans MURRAY, A History of Chess (voir n. 1), et dans les ouvrages abondamment illustrés cités à la note 20 . 